2014-06

\title{
Destructive behavior in a Fragile Public Good game
}

Hoyer, Maximilian

http://hdl.handle.net/10026.1/15297

10.1016/j.econlet.2014.02.023

Economics Letters

Elsevier BV

All content in PEARL is protected by copyright law. Author manuscripts are made available in accordance with publisher policies. Please cite only the published version using the details provided on the item record or document. In the absence of an open licence (e.g. Creative Commons), permissions for further reuse of content should be sought from the publisher or author. 


\title{
Destructive behavior in a Fragile Public Good game
}

\author{
Maximilian Hoyer ${ }^{\mathrm{a}, \mathrm{b}, *}$, Nadège Bault ${ }^{\mathrm{a}, \mathrm{b}, \mathrm{c}}$, Ben Loerakker ${ }^{\mathrm{a}, \mathrm{b}}$, Frans van Winden ${ }^{\mathrm{a}, \mathrm{b}}$ \\ ${ }^{\text {a }}$ University of Amsterdam, Faculty of Economics and Business, CREED, Roeterstraat 11, 1018 WB Amsterdam, The Netherlands \\ ${ }^{\mathrm{b}}$ Cognitive Science Center Amsterdam, University of Amsterdam, Nieuwe Achtergracht 129, 1018 Ws Amsterdam, The Netherlands \\ ${ }^{\mathrm{c}}$ Groupe d'Analyse et de Thorie Economique (GATE), CNRS and University of Lumiere Lyon 2, 69130 Ecully, France
}

\section{H I G H L I G H T S}

- Substantial destructive behavior observed in a Fragile Public Good environment.

- Destructive behavior is modulated by the specific nature of the environment.

- Equilibration towards both cooperative and sour (spiteful) relationships observed.

- Framing and subject characteristics can affect the incidence of destructive behavior.

\section{A R T I C L E I N F O}

\section{Article history:}

Received 31 October 2013

Received in revised form

19 February 2014

Accepted 28 February 2014

Available online 6 March 2014

\section{JEL classification:}

C9

$\mathrm{H} 4$

\section{Keywords:}

Public good

Destructive behavior

Spite

Relationship

Laboratory experiment

\begin{abstract}
A B S T R A T
Socially destructive behavior in a public good environment - like damaging public goods - is an underexposed phenomenon in economics. In an experiment we investigate whether such behavior can be influenced by the very nature of an environment. To that purpose we use a Fragile Public Good (FPG) game which puts the opportunity for destructive behavior (taking) on a level playing field with constructive behavior (contributing). We find substantial evidence of destructive decisions, sometimes leading to sour relationships characterized by persistent hurtful behavior. While positive framing induces fewer destructive decisions, shifting the selfish Nash towards minimal taking doubles its share to more than $20 \%$.
\end{abstract}

(C) 2014 Elsevier B.V. All rights reserved.

\section{Introduction}

Many experimental studies have investigated the development of cooperation in a social dilemma or public good environment, and the effect of punishment mechanisms in this context (for a recent survey, see Chaudhuri, 2011). In the real world, however, people can often cooperate with or hurt one another. Relationships may even turn sour and induce persistent destructive behavior. Repeated and severe conflict is a real part of human interaction. Examples are neighborhood conflicts, family feuds, or the destruction of public property during riots. In some studies, a substantial proportion of individuals engaged in the destruction of others' earnings, even when rank egalitarianism and reciprocity motives were not present and when the destruction was costly (Zizzo, 2003; Abbink and Sadrieh, 2009). To study whether destructive behavior

\footnotetext{
* Correspondence to: University of Amsterdam, CREED, Roeterstraat 11, 1018 WB Amsterdam, The Netherlands. Tel.: +31 205254178; fax: +31 634418749.

E-mail address: m.o.hoyer@uva.nl (M. Hoyer).
}

can be observed and modulated in a public good environment we designed a 'Fragile Public Good' (FPG) game. A key feature of the FPG game is that it gives as much room for destructive behavior (taking) as for constructive behavior (contributing). More formally, it does so by shifting both the (standard) Nash equilibrium and the status quo - i.e., the initial allocation of tokens to the common account - to the middle of the action space, with perfect symmetry in the marginal cost of taking and contributing. Contrary to the relatively few public good experiments that allow for an interior Nash equilibrium (see surveys by Laury and Holt, 2008 and Saijo, 2008), we focus on destructive actions in a repeated context where subjects can identify the individual decisions of others.

This paper is related to a developing stream of literature on 'feuds' (Nikiforakis and Engelmann, 2011; Nikiforakis et al., 2012) and 'vendettas' (Fehl et al., 2012; Abbink and Herrmann, 2009; Bolle et al., forthcoming). These experiments typically focus strongly on the punishment by explicitly separating contribution and punishment stages. For example, Nikiforakis and Engelmann (2011) use separate punishment rounds after a 4-player public 
Table 1

Average contributions.

\begin{tabular}{llrl}
\hline & SYM & \multicolumn{1}{l}{ FRAME } & \multicolumn{1}{l}{ ASYM } \\
\hline Overall & $2.28(2.01)$ & $2.07(1.69)$ & $1.83(3.3)$ \\
First round & $1.26(2.55)$ & $-0.02(2.55)$ & $0.92(3.06)$ \\
Rounds 26-34 & $2.44(2.35)$ & $2.49(3.85)$ & $2.13(3.85)$ \\
Last round (35) & $0.68(2.43)$ & $0.98(1.81)$ & $0.31(3.81)$ \\
\hline
\end{tabular}

Note: adjusted for the (standard) Nash equilibrium; standard deviation in parentheses, with dyad averages as separate observations.

good game, whereas Bolle et al. (forthcoming) let subjects decrease others' probabilities of winning a prize.

Because framing can influence behavior in public good games (Brewer and Kramer, 1986; Sonnemans et al., 1998; Willinger and Ziegelmeyer, 1999; for a survey, see Cookson, 2000), we study the sensitivity of our findings in two additional treatments, where we separate the status quo from the Nash outcome. In one case, we move the status quo to a corner so that subjects can only contribute, keeping everything else the same; this is a case of positive framing which may induce subjects to contribute more. In the other case, we move the Nash outcome away from the status quo towards taking by introducing a slight payoff asymmetry. Here the Nash choice may be read as aggression by subjects using the status quo as a reference point and induce destructive behavior.

Our main questions are: (1) does the FPG game generate destructive behavior and even cases where behavior equilibrates towards sour relationships? (2) How does separating the Nash outcome from the status quo through framing or some minimal payoff asymmetry modulate taking and contributing? After the design we present our results, followed by a summary of our findings.

\section{Experiment}

Subjects played the FPG game in fixed dyads over 35 rounds in all three treatments.

\subsection{Symmetric treatment (SYM)}

In each round both subjects of a dyad are endowed with a private account holding 7 tokens, earning 10 units each, and a common account holding 14 tokens, earning 10 each for both subjects. Subjects can contribute to or take up to 7 tokens from the common account, at increasing marginal costs: moving one token costs 2 units, while the marginal transfer cost of each additional token increases by $2 .{ }^{1}$ Earnings are symmetric around the status quo which coincides with the selfish Nash outcome, while any combination of contributions of 4 or 5 is socially optimal.

\subsection{Framing treatment (FRAME)}

In FRAME subjects have exactly the same strategy space and equivalent earnings, but now they start each round with 14 tokens in their private accounts and the common account is empty. Thus, to reach an outcome equivalent to an outcome in SYM, subjects would have to contribute 7 more tokens than before. ${ }^{2}$ Because only contributions can be made, this is a case of positive framing.

\subsection{Asymmetric treatment (ASYM)}

ASYM differs from SYM in only two respects: tokens in the private account earn subjects 11 units instead of 10 , and the first

\footnotetext{
1 Formally, we use the following payoff function, where $c_{i}$ can be positive or negative: $V_{A}\left(c_{A}, c_{B}\right)=10\left(14+c_{A}+c_{B}\right)+10\left(7-c_{A}\right)-\left(\left|c_{A}\right|+c_{A}^{2}\right)$. See Fig. 5 in the Appendix.

2 Payoff function: $V_{A}\left(c_{A}, c_{B}\right)=10\left(c_{A}+c_{B}\right)+10\left(14-c_{A}\right)-\left(\left|c_{A}-7\right|+\left(c_{A}-7\right)^{2}\right)$.
}

token transferred in either direction has zero costs. As in FRAME, the Nash equilibrium does not coincide with the status quo, but now it is the former that moves by prescribing to take one token out of the common account, while both subjects contributing 5 tokens is the social optimum. ${ }^{3}$

Subjects did not see the underlying formulas, but were supplied with graphs illustrating the marginal effects of every decision for themselves and the other, alongside with payoff tables. ${ }^{4}$

The public good game was preceded by a test of social value orientation (SVO; see Liebrand and McClintock, 1988, taken from Van Dijk et al., 2002). This test measures the preferences of subjects for distribution outcomes for themselves and a (generalized) other. Sessions were run in November and December 2012 and April 2013 at the CREED-lab in Amsterdam. SYM had 130 participants (50\% female, 2\% unreported gender, average age 22.2), FRAME 54 (41\% female, average age 21.5), and ASYM 80 (43\% female, average age 21.5). The experiment had an additional second part, which we do not cover in this paper. The exchange rate of units into euros was 700 to one. Subjects earned on average 1.45 euro in the SVO-test and 10.82 euro in the public good game.

\section{Results}

Table 1 gives an overview of average contributions, where we adjust for the Nash equilibrium (NE) in each game by subtracting 7 tokens from results in FRAME and adding 1 to results in ASYM.

Average contributions are approximately 2 tokens above the Nash-prediction in all treatments. The first round, however, reveals a different pattern as the average contribution in FRAME is significantly lower than in SYM $(p=0.001) .{ }^{5}$ Because SYM and ASYM are more similar to a taking game than FRAME (where only contributions are possible), this result contrasts with the general finding that there are typically lower contributions in taking framings, if there is any difference (Andreoni, 1995; Sonnemans et al., 1998; Goerg and Walkowitz, 2010). Khadjavi and Lange (2013) have a treatment with intermediate endowments similar to our SYM and ASYM treatments and find no differences between a contributing frame and this alternative.

Subjects appear to be reluctant to contribute early on in FRAME, but are able to compensate for this throughout the game, as the difference stays significant at $1 \%$ up until the fifth round of the game.

All treatments show an increase in contributions over time until the (usually observed) sharp decline at the end; see Fig. $1 .^{6} \mathrm{~A}$ simple regression shows significant positive time trends. Although the increase is at odds with the general observation of decreasing cooperation in public good experiments (Ledyard, 1995), it has been observed before in repeated two-player games using a comparable mechanism (Van Dijk et al., 2002). Comparing SYM and ASYM, the hypothesis of equal contributions is rejected if they are calculated relative to the status quo ( $p=0.035$ ), but not relative to the Nash equilibrium outcome, which may suggest that the latter is a more important reference point.

Most relevant to this paper is the occurrence and development of destructive behavior hurting both partners. Relative to all decisions, destructive decisions count 11\% in SYM, 7\% in FRAME, and $21 \%$ in ASYM; see Table 2. The percentages of subjects choosing below the Nash at least once are, respectively, $42 \%, 46 \%$, and $56 \%$. The higher number of destructive decisions in ASYM, despite similar average contribution levels relative to the Nash outcome (see

\footnotetext{
3 Payoff function: $V_{A}\left(c_{A}, c_{B}\right)=10\left(14+c_{A}+c_{B}\right)+11\left(7-c_{A}\right)+\left|c_{A}\right|-c_{A}^{2}$.

4 Instructions are available upon request.

5 We use the Mann-Whitney $U$-test with dyad averages as observations unless otherwise mentioned.

6 Contributions in the last round are lower than in the ten rounds before ( $p<$ 0.01 in all treatments).
} 


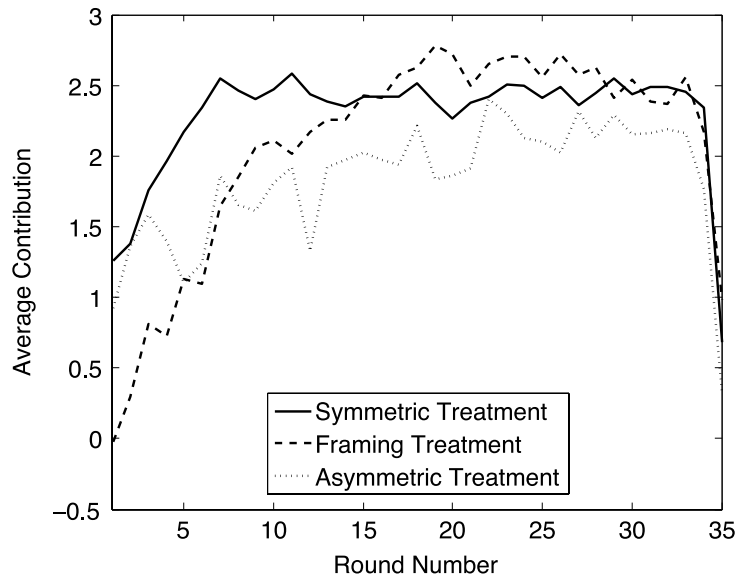

Fig. 1. Average contributions, relative to Nash equilibrium.

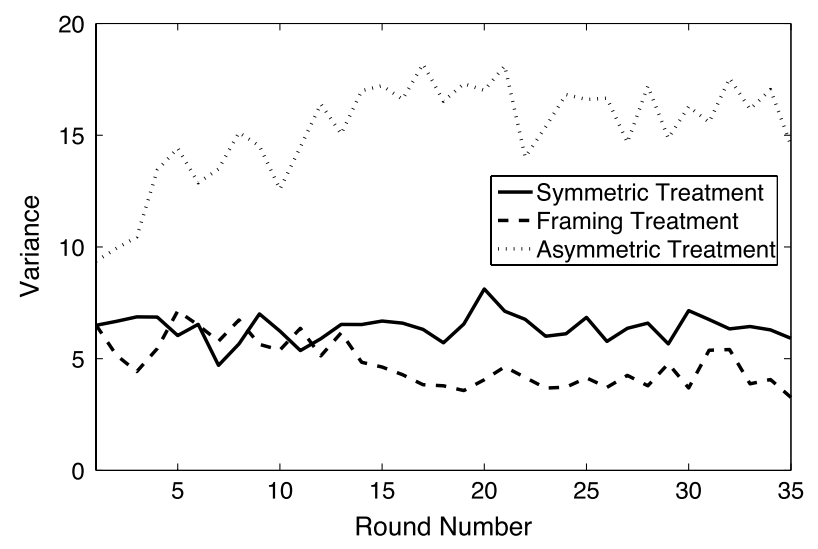

Fig. 2. Between-subject variance across treatments/rounds.

Table 2

Percentage of destructive decisions.

\begin{tabular}{llll}
\hline & SYM & FRAME & ASYM \\
\hline Overall & $11.25 \%$ & $6.93 \%$ & $21.14 \%$ \\
Last round & $13.9 \%$ & $5.36 \%$ & $25 \%$ \\
\hline
\end{tabular}

Table 1), suggests distributional differences. Indeed, the variance of subjects' decisions is larger in ASYM than in SYM and FRAME in 31 of the 35 rounds (Levene's test, $p<0.01$; see also Fig. 2). Interestingly, this difference only becomes significant from the third round onwards, which indicates that it is partly driven by the dynamics in the game. Not only the variance across subjects, but also the variance within each subject's set of 35 decisions is greater in ASYM. ${ }^{7}$ Summing the destructive decisions of each dyad we find a difference only between SYM and ASYM $(p=0.094) .{ }^{8}$ The higher level of conflict observed in ASYM is confirmed by the observation that the percentage of destructive decisions in the last round (when there are no strategic considerations present) is higher in ASYM than in the other treatments.

Background measures seem to play a role in explaining destructive behavior: In ASYM and SYM contributions are lower and destruction rates higher in female dyads than in male dyads at $5 \%$ significance levels and SVO correlates with individual decisions. ${ }^{9}$

\footnotetext{
7 Means of within-subject variances: 2.57 in SYM, 2.54 in FRAME, and 4.59 in ASYM. The differences between SYM and ASYM $(p<0.001)$ and FRAME vs ASYM $(p=0.038)$ are significant.

8 In line with a result of Nikiforakis et al. (2012), who find higher rates of counterpunishment in a treatment with increased normative conflict.

9 In ASYM with $p \leq 1 \%$ for contributions in the first round, first 5 rounds, and the whole game, and (negatively) with the number of destructive decisions; $p \leq 5 \%$ for
}

Table 3

Probabilities of continuing a feud.

\begin{tabular}{ll}
\hline Feud length & Ratio (\%) \\
\hline 1 & $51 / 111(45.9 \%)$ \\
2 & $26 / 50(52.0 \%)$ \\
3 & $19 / 26(73.1 \%)$ \\
4 & $16 / 19(84.2 \%)$ \\
5 & $13 / 15(86.7 \%)$ \\
6 & $10 / 13(76.9 \%)$ \\
7 & $7 / 10(70.0 \%)$ \\
$8+$ & $75 / 76(98.7 \%)$ \\
\hline
\end{tabular}

Table 4

Number of distinct dyads with at least one feud of a certain length.

\begin{tabular}{lllll}
\hline Feud length & SYM & FRAME & ASYM & Total \\
\hline Feuds of any length & $16(52)$ & $4(6)$ & $18(57)$ & $38(115)$ \\
Length $=1$ & $14(29)$ & $3(5)$ & $16(30)$ & $33(64)$ \\
Length $=2$ & $9(13)$ & $0(0)$ & $7(12)$ & $16(25)$ \\
\hline
\end{tabular}

Note: first number reflects distinct dyads that are involved in feuds. Overall number of feuds in parentheses.

It appears that sour relationships do indeed develop in the FPG game, as illustrated in two examples in Fig. 3.

The most restrictive definition of a sour relationship is to only consider instances of mutual destruction by both members of a dyad at the same time. Fig. 4 shows these instances and separates them by the number of rounds that they survive, linking up with the feud-literature (Nikiforakis and Engelmann, 2011; Nikiforakis et al., 2012). A majority of such relationships only survives for one period, but a total of 51 develops past the first round. Since the experiment ended after 35 rounds we report the cases in which conflict is cut off at the end separately. Table 3 further shows the probability with which mutual destructiveness proceeds after different numbers of rounds, now denoted as feuds. We see that the cases which survive past the first round have increasing probabilities of proceeding further. Table 4 reports the number of distinct dyads that face at least one feud. We also ran a regression of the probability of mutual punishment in the aggregate data using a dyad-level logit model with random effects and using dummies indicating a past series of exactly one, two, or three - but not more and four or more consecutive cases of mutual destruction in each dyad, together with two similar variables for only one subject being destructive as explanatory variables. All these coefficients are highly significant, those for both having been destructive are bigger than those for only one having been destructive, and they increase in size from two consecutive rounds onwards. ${ }^{10}$

\section{Conclusion}

This study shows that substantial destructive behavior can occur even in a public good environment once the opportunity to do so is present. Our baseline Fragile Public Good game - offering players equal room to take from or contribute to a public good, against symmetric marginal costs - showed more than $10 \%$ destructive decisions. While, unexpectedly, positive framing had significant negative effects on contributing in the early rounds of the game, players compensated for that later on, such that on average fewer

the first round in SYM; $p \leq 5 \%$ for the first 5 rounds and average contribution in FRAMING, and $p \leq 10 \%$ for the first round and the overall number of destructive decisions.

10 Coefficients between 4.4 and 6.9 (s.e. between 0.47 and 0.65 ). This specification is problematic due to the dynamic nature of the regressors, which we try to tackle by using variables that are unique within a feud. Alternative approaches, including a subject-level based random effects model with additional explanatory variables and with subjects nested in dyads as random effects produced qualitatively similar results in that longer running periods of mutual destruction generally led to higher increases in the probability of feud progression. 

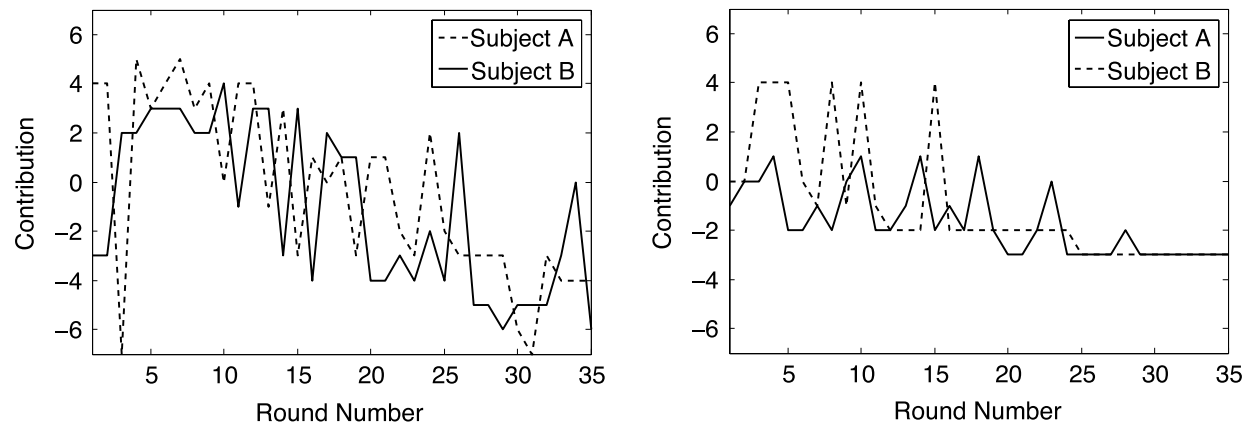

Fig. 3. Examples of sour relationships in SYM.

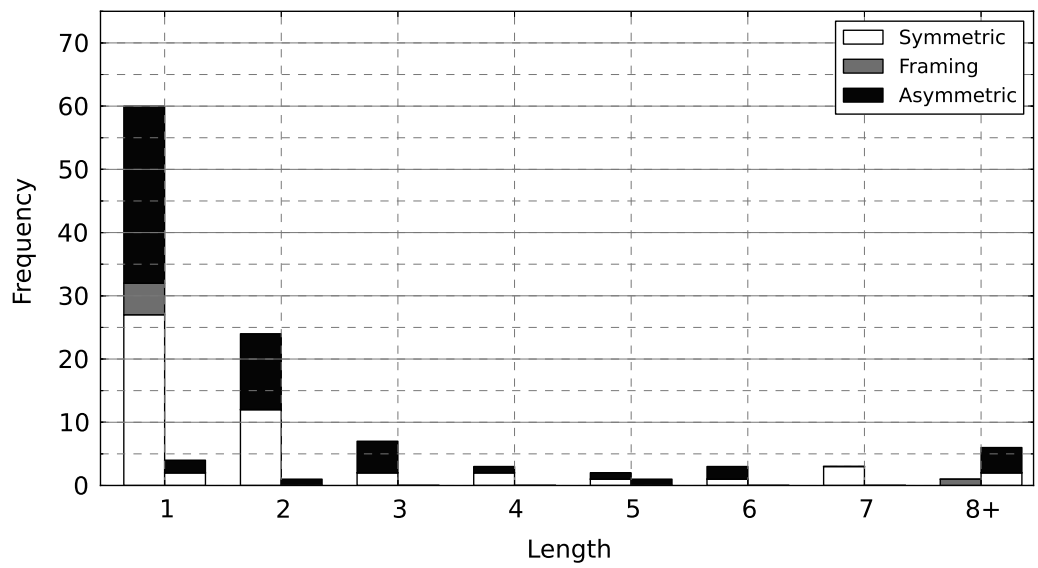

Fig. 4. Distribution of feuds that end before (left bar) and at (right bar) the final round.

destructive decisions were observed. Introducing a slight asymmetry by separating the Nash outcome from the initial status quo towards taking one token sharply increased the share of destructive decisions to more than $20 \%$ (even in the last round). Finally, we show that feuds can occur in a setting without separate punishment and it becomes increasingly difficult to exit them as they last longer.

\section{Acknowledgments}

This paper is part of the Research Priority Program Brain \& Cognition at the University of Amsterdam. Financial support by the Research Priority Area Behavioral Economics of the University of Amsterdam (201303180403), the French National Research Agency (ANR-11-EMCO-01101) and the LABEX CORTEX (ANR-11LABX-0042) is gratefully acknowledged.

The authors would like to thank Benjamin Pelloux, at the time affiliated with CREED and CSCA, for his contribution to the initial design of this project. They are further thankful for helpful comments by attendees of the Economic Science Association Annual Meeting 2013 and the Tilburg Institute for Behavioral Economics Research conference 2013 and an anonymous referee.

\section{Appendix}

See Fig. 5.

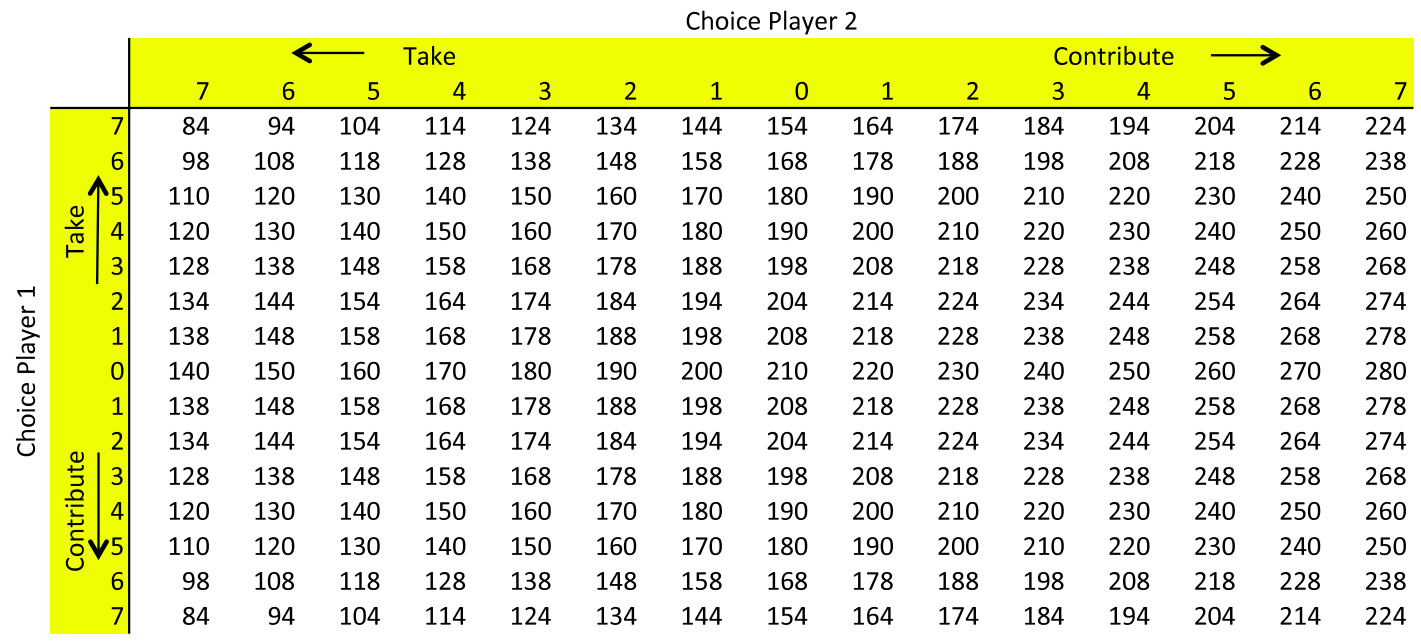

Fig. 5. Earnings of player 1 , SYM. 


\section{References}

Abbink, K., Herrmann, B., 2009. Pointless vendettas. Norwich: University of East Anglia CBESS Working Paper.

Abbink, K., Sadrieh, A., 2009. The pleasure of being nasty. Econom. Lett. 105 (3), 306-308.

Andreoni, J., 1995. Warm-glow versus cold-prickle: the effects of positive and negative framing on cooperation in experiments. Quart. J. Econ. 110 (1), 1-21.

Bolle, F., Tan, J., Zizzo, D., 2013. Vendettas. Amer. Econ. J.: Microecon. (forthcoming) Brewer, M.B., Kramer, R.M., 1986. Choice behavior in social dilemmas: effects of social identity, group size, and decision framing. J. Pers. Soc. Psychol. 50 (3), 543-549.

Chaudhuri, A., 2011. Sustaining cooperation in laboratory public goods experiments: a selective survey of the literature. Exp. Econ. 14 (1), 47-83.

Cookson, R., 2000. Framing effects in public goods experiments. Exp. Econ. 3 (1), 55-79.

Fehl, K., Sommerfeld, R.D., Semmann, D., Krambeck, H.-J., Milinski, M., 2012. dare you to punish me-vendettas in games of cooperation. PLoS One 7 (9) e45093.

Goerg, S.J., Walkowitz, G., 2010. On the prevalence of framing effects across subjectpools in a two-person cooperation game. J. Econ. Psychol. 31 (6), 849-859.

Khadjavi, M., Lange, A., 2013. Doing good or doing harm-experimental evidence on giving and taking in public good games. Technical Report, Working Paper.
Laury, S.K., Holt, C.A., 2008. Voluntary provision of public goods: experimental results with interior Nash equilibria. In: Plott, C.R., Smith, V.L. (Eds.), Handbook of Experimental Economics Results, vol. 1. Elsevier, Amsterdam, pp. 792-801.

Ledyard, J., 1995. Public goods: a survey of experimental research. In: Kagel, J. Roth, A.E. (Eds.), Handbook of Experimental Economics. Princeton University Press, Princeton, pp. 111-181.

Liebrand, W., McClintock, C., 1988. The ring measure of social values: a computerized procedure for assessing individual differences in information processing and social value orientation. Eur. J. Pers. 2, 217-230.

Nikiforakis, N., Engelmann, D., 2011. Altruistic punishment and the threat of feuds. J. Econ. Behav. Organ. 78 (3), 319-332.

Nikiforakis, N., Noussair, C.N., Wilkening, T., 2012. Normative conflict and feuds: the limits of self-enforcement. J. Public Econ. 96 (9), 797-807.

Saijo, T., 2008. Spiteful behavior in voluntary contribution mechanism experiments. In: Plott, C.R., Smith, V.L. (Eds.), Handbook of Experimental Economics Results, vol. 1. Elsevier, Amsterdam, pp. 802-816.

Sonnemans, J., Schram, A., Offerman, T., 1998. Public good provision and public bad prevention: the effect of framing. J. Econ. Behav. Organ. 34 (1), 143-161.

Van Dijk, F., Sonnemanns, J., van Winden, F., 2002. Social ties in a public good experiment. J. Public Econ. 85, 275-299.

Willinger, M., Ziegelmeyer, A., 1999. Framing and cooperation in public good games: an experiment with an interior solution. Econom. Lett 65 (3), 323-328.

Zizzo, D.J., 2003. Money burning and rank egalitarianism with random dictators. Econom. Lett. 81 (2), 263-266. 